\title{
EFAS Score - Validation of Finnish and Turkish Versions by the Score Committee of the European Foot and Ankle Society (EFAS)
}

\section{Richter, Martinus}

2020-04

Richter , M , Agren , P-H , Besse , J-L , Coester , M , Kofoed , H, Maffulli , N , Steultjens , M , Irgit , K , Miettinen , M , Repo , J P \& Uygur , E 2020 , ' EFAS Score - Validation of Finnish and Turkish Versions by the Score Committee of the European Foot and Ankle Society (EFAS) ' , Foot and Ankle Surgery , vol. 26 , no. 3 , pp. 250-253 . https://doi.org/10.1016/j.fas.2020.03.004

http://hdl.handle.net/10138/327959

https://doi.org/10.1016/j.fas.2020.03.004

cc_by_nc_nd

acceptedVersion

Downloaded from Helda, University of Helsinki institutional repository.

This is an electronic reprint of the original article.

This reprint may differ from the original in pagination and typographic detail.

Please cite the original version. 


\section{Journal Pre-proof}

EFAS Score - Validation of Finnish and Turkish Versions by the Score Committee of the European Foot and Ankle Society (EFAS)

Martinus Richter Per-Henrik Agren Jean-Luc Besse Maria Coester Hakon Kofoed Nicola Maffulli Martijn Steultjens Kaan Irgit Mikko Miettinen Jussi P. Repo Esat Uygur

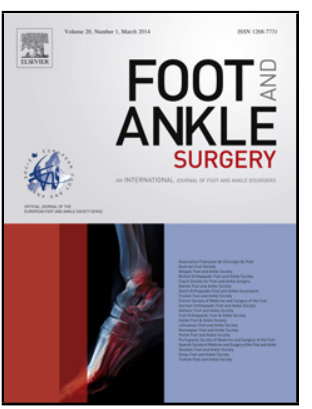

PII:

S1268-7731(20)30042-4

DOI: https://doi.org/doi:10.1016/j.fas.2020.03.004

Reference: FAS 1428

To appear in: Foot and Ankle Surgery

Please cite this article as: Richter M, Agren P-H, Besse J-L, Coester M, Kofoed H, Maffulli N, Steultjens M, Irgit K, Miettinen M, Repo JP, Uygur E, EFAS Score - Validation of Finnish and Turkish Versions by the Score Committee of the European Foot and Ankle Society (EFAS), Foot and Ankle Surgery (2020), doi: https://doi.org/10.1016/j.fas.2020.03.004

This is a PDF file of an article that has undergone enhancements after acceptance, such as the addition of a cover page and metadata, and formatting for readability, but it is not yet the definitive version of record. This version will undergo additional copyediting, typesetting and review before it is published in its final form, but we are providing this version to give early visibility of the article. Please note that, during the production process, errors may be discovered which could affect the content, and all legal disclaimers that apply to the journal pertain.

(C) 2020 Published by Elsevier. 
1 EFAS Score - Validation of Finnish and Turkish Versions by the

2 Score Committee of the European Foot and Ankle Society

3 (EFAS)

4

Martinus Richter ${ }^{\mathrm{a}, 1}$, Per-Henrik Agren ${ }^{\mathrm{b}, 2,{ }^{*}}$, Jean-Luc Besse ${ }^{\mathrm{b}, 3,{ }^{*}}$, Maria Coester ${ }^{\mathrm{b},}$

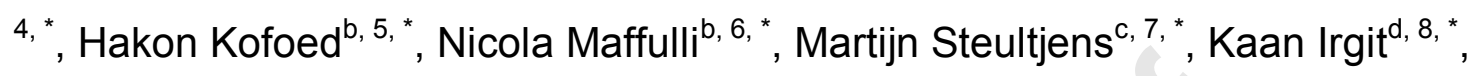

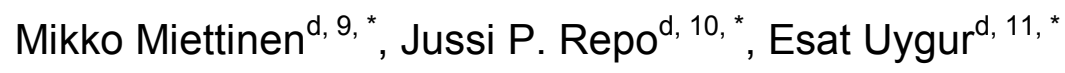

${ }^{a-d}$ Score Committee European Foot and Ankle Society, c/o European Foot and Ankle Society (EFAS), Brussels, Belgium

a

Head and core member

b Core member

c Outcome measure development expert and core member

d National affiliate member

Equal coauthor

1 Department for Foot and Ankle Surgery Rummelsberg and Nuremberg, Schwarzenbruck, Germany

2 Stockholms Fotkirurgklinik, Sophiahemmet University, Stockholm, Sweden

3 Laboratoire de Biomecanique et Mecanique des Chocs, Universite Lyon, Bron Cedex, France and Service de Chirurgie Orthopedique et Traumatologique, Hospices Civils de Lyon, Centre Hospitalier Lyon-Sud, Pierre-Benite Cedex, France.

4 Department of Clinical Sciences and Orthopedics, Skane University Hospital, Malmoe, Sweden and Department of Foot and Ankle Surgery, Capio Movement, Halmstad, Sweden Charlottenlund, Denmark

6 Queen Mary University of London, Barts and The London School of Medicine and Dentistry, London, UK 
7 School of Health and Life Sciences, Glasgow Caledonian University, Glasgow, Scotland, UK

8

Department of Orthopaedics and Traumatology, Pendik Training and

Research Hospital, Marmara University, Istanbul, Turkey

9 Department of Orthopedics and Traumatology, Peijas Hospital, Helsinki

University Hospital and University of Helsinki, Helsinki, Finland

10

Department of Orthopedics and Traumatology, Central Finland Health Care

District, Jyvaeskylae, Finland

${ }^{11}$ Department of Orthopaedics and Traumatology, Goztepe Training and

Research Hospital, Istanbul Medeniyet University, Istanbul, Turkey

Corresponding author:

Score Committee European Foot and Ankle Society (EFAS)

European Foot and Ankle Society (EFAS)

280, Boulevard du Souverain

1160 Brussels

Belgium

7 Abstract

8 Background

9 The Score Committee of the European Foot and Ankle Society (EFAS)

10 developed, validated, and published the EFAS Score in seven European

11 languages (English, German, French, Italian, Polish, Dutch, Swedish). From

12 other languages under validation, the Finnish and Turkish versions finished data

13 acquisition and underwent further validation.

14 Methods 
15 The EFAS Score was developed and validated in three stages: 1) item

16 (question) identification (completed during initial validation study), 2) item

17 reduction and scale exploration (completed during initial validation study), 3)

18 confirmatory analyses and responsiveness of Finnish and Turkish version

19 (completed during initial validation study in seven other languages). The data

20 were collected pre-operatively and post-operatively at a minimum follow-up of 3

21 months and mean follow-up of 6 months. Item reduction, scale exploration,

22 confirmatory analyses and responsiveness were executed using classical test

23 theory and item response theory.

24 Results

25 The internal consistency of the scale was confirmed in the Finnish and Turkish

26 versions (Cronbach's Alpha >0.8). Responsiveness was good, with moderate to

27 large effect sizes in both languages, and evidence of a statistically significant

28 positive association between the EFAS Score and patient-reported improvement.

\section{Conclusions}

30 The Finnish and Turkish EFAS Score versions were successfully validated in the

31 orthopaedic ankle and foot surgery patients, including a wide variety of foot and

32 ankle pathologies. All score versions are freely available at www.efas.co.

\section{Keywords}

35 Score; Foot; Ankle; Validation; PROM

\section{Introduction}

38 The Score Committee of the European Foot and Ankle Society (EFAS)

39 developed, validated, and published the EFAS Score in seven European

40 languages (English, German, French, Italian, Polish, Dutch, Swedish)[1]. The 
41 score covers pain and physical function. The EFAS Score is internally consistent,

42 unidimensional and responsive to change in samples of orthopaedic foot and

43 ankle surgery patients[1]. The score contains six questions. The maximum score

44 is 24 points (best possible), and the minimum 0 points (worst possible). The

45 language-specific cross-cultural validation was necessary because simple

46 translation of a validated score does not necessarily result in an instrument that

47 provides valid scores in the target language[1]. This issue is especially important

48 for Europe with numerous languages[1]. The most spoken mother tongues in

49 Europe are German (16\%), English (13\%), Italian (13\%), French (12\%), Spanish

50 (8\%), Polish (8\%), Romanian (5\%) and Dutch (4\%) (source Wikipedia, January

51 16, 2020). Therefore, a need for different language-specific (validated) scores,

52 especially in Europe, is clear[1]. After having validated the EFAS Score in seven

53 languages initially, the data acquisition in eight other languages (Arabic, Danish,

54 Finnish, Hungarian, Norwegian, Portuguese, Spanish, Turkish) started. This data

55 acquisition was finished in Finnish and Turkish so far and the results of the

56 validation process and the results scores are presented.

\section{Methods}

59 The EFAS patient-reported outcome measure (PROM), the 'EFAS Score', was

60 developed and validated in three stages: 1) item identification, 2) item reduction

61 and scale exploration, 3) confirmatory analyses and responsiveness[1].

63 Type of score (initial score development)[1].

64 A questionnaire-based PROM, with a 5-point Likert scale (0-4) was chosen[1].

66 Questions - Item identification (initial score development)[1]. 
67 In the first stage of the initial validation, potentially relevant items from existing

68 questionnaires were identified[1]. Given the low relevance of items related to

69 sports activities for some diagnostic groups, it was decided at this point to

70 develop two separate scores: a general item score and a sports-specific score[1].

71 In total, 31 general items and 7 sports-specific items were taken forward into the

72 second phase of the project[1].

73

74 Item reduction and scale exploration (initial score development)[1].

75 Through a process of forward and backward translation performed by bilingual

76 translators, the original English pool of 38 items was translated into German,

77 French and Swedish[1]. These four language versions were then used for the

78 Stage 2 data collection[1]. Participants were recruited from orthopaedic foot and

79 ankle surgery departments[1]. Inclusion criteria for participants were clinical and

80 imaging indications for foot and ankle surgery and age $\geq 18$ years[1]. No

81 exclusion criteria were used other than an inability to complete a written

82 questionnaire[1]. Data collection was performed in France, Germany, Sweden

83 and Ireland[1]. In addition to providing an answer to each item on a 5-point

84 scale, all participants also rated the relevance of the item to their situation on a 5-

85 point scale[1].

86

87 Following data collection, the following analytic steps were taken to reduce the

88 item pool into one general PROM and one sports PROM[1].

89 1. Items with a ceiling effect, low perceived relevance and a high proportion of

90 missing values were noted and shortlisted for exclusion in subsequent

91 steps[1]. 
92 2. A principal component analysis (PCA) was performed[1]. At the end of this

93 step, the remaining items in their respective principal components would

94 provide optimal scale reliability according to classic test theory[1].

95 3. An Item-response theory (IRT) analysis was performed for each of the 96 identified scales (i.e., principal components) to further reduce the number of $97 \quad$ items and optimize scale unidimensional[1].

99 Confirmatory analysis and responsiveness (initial score validation)[1]

100 Data collection for this final stage of the initial validation took place in the four

101 original language versions, as well as Dutch, Italian and Polish[1].

103 Confirmatory analysis and responsiveness Finnish and Turkish versions

104 Data collection stage of the validation was performed in Finland and Turkey.

105 Inclusion criteria for participants were scheduled foot and ankle surgery and age

$106 \geq 18$ years. No exclusion criteria were used other than an inability to complete a

107 written questionnaire. Data were collected preoperatively and at postoperative

108 follow-up. Minimum postoperative follow-up of 3 months and mean follow-up of 6 109 months planned, collecting at least 100 completed score sheets. To confirm the

110 internal consistency for each language version, Cronbach's Alpha of the EFAS

111 Score was computed for each language version separately[1]. To establish the

112 responsiveness of the EFAS Scores, both distribution-based and criterion-based

113 analyses were used[1]. Distribution-based measures of responsiveness included

114 the effect size (ES) and minimal important difference (MID)[1]. The criterion-

115 based measure of responsiveness used was the linear association (Pearson's

116 correlation) between improvement on the EFAS Score and a 5-point Likert scale 
117 anchor question: did the surgery improve the foot and/or ankle problem? $(0=$ no,

118 not at all; $4=$ yes, very much)[1].

119 The ES was calculated as the difference between the baseline and three to six-

120 month follow-up mean EFAS Score, divided by the standard deviation of the

121 baseline EFAS Score[1].

122 The MID was considered to be equal to the standard error of measurement

123 (SEM) of the baseline EFAS Score. The SEM was calculated as[1]:

124

$125 S E M=S D * \sqrt{1-r} \quad$ (Formula 1), where:

126

127 SD = standard deviation of the EFAS Score baseline score

$128 \mathrm{r}=$ value of Cronbach's Alpha for the EFAS Score at baseline.

130 To assess the responsiveness of the EFAS Score using the MID, the percentage

131 of participants with an improvement in their EFAS Score between baseline and

132 follow-up exceeding the MID was identified[1].

134 Statistical analyses were performed in SPSS (IBM SPSS Statistics 23, IBM,

135 Armonk, NY, USA). The IRT modelling was performed in XCalibre 4 (Assessment

136 Systems, Inc.)

138 Ethics

139 Approvals from the relevant ethical committees in different contributing countries

140 were obtained, adhering to local legislation. 


\section{Results}

144 Table 1 and 2 show the language-specific demographic data (Table 1) and

145 diagnoses (Table 2) for the patient samples.

147 Confirmatory analyses and responsiveness

148 The internal consistency of the scale was excellent in both language versions.

149 Cronbach's Alpha was 0.84 in Finnish and 0.81 in Turkish. Responsiveness of

150 the EFAS Score is shown in Table 3 and Figures 1a and b. Large effect sizes

151 (ES>0.8) were found in both language versions. A clear majority of patients

152 showed a minimally important difference following surgery, $67.7 \%$ in Finnish and

$15379.4 \%$ in Turkish. The change in EFAS Scores between baseline and follow-up

154 was significantly correlated with the patient-reported change in health status.

156 Discussion

157 The EFAS Score was successfully validated in Finnish and Turkish. Not all

158 measurement properties of the EFAS Score have been established. In particular

159 test-retest reliability, i.e. reproducibility of the score in a stable (pre-surgery)

160 population, was not included in the initial validation and the present study[1]. The

161 MID as reported in this and the initial validation study was based on the internal

162 consistency of the scale (Cronbach's Alpha) rather than test-retest reliability[1]. In

163 future, if the test-retest reliability becomes available, this may lead to an

164 adjustment in the SEM and therefore MID of the EFAS Score.

165 The process to develop the EFAS Sports Score was ultimately unsuccessful

166 during the initial validation study[1]. The questions related to sports activities

167 were not relevant to a large proportion of the patient samples, and suffered from

168 a high proportion of missing values[1]. This implies that the IRT modelling did not 
169 result in a unidimensional EFAS Sports Score[1]. Based on the findings of the

170 IRT model, a 4-item EFAS Sports Score could be considered, as this was the

171 best-performing option[1]. The EFAS Sports Score was included in the data

172 acquisition of all languages because this was part of the initially defined

173 validation process that was decided not be changed during the process[1].

175 In conclusion, the Finnish and Turkish EFAS Score versions were successfully

176 validated in the orthopaedic ankle and foot surgery patient population, including a

177 wide variety of foot and ankle pathologies. All score versions are freely available

178 at www.efas.co.

179

180 Acknowledgements

181 The EFAS Score Committee thanks the following contributors for data

182 acquisition: Antti Latvala, Department of Orthopedics and Traumatology, Oulu

183 University Hospital, Oulu, Finland; Alar Toom, Department of Surgery, Central

184 Finland Health Care District, Jyvaeskylae, Turkey; Emre Baca and M. Utku Ciftci,

185 Orthopaedics and Traumatology, Bakirkoy Sadi Konuk Training and Research

186 Hospital, Istanbul, Turkey; Omer Büyüktopcu, Orthopaedics and Traumatology,

187 Marmara University, Faculty of Medicine, Istanbul, Turkey.

190 Reference

191 1. Richter M, Agren PH, Besse JL, Coester M, Kofoed H, Maffulli N,

192 Rosenbaum D, Steultjens M, Alvarez F, Boszczyk A, Buedts K, Guelfi M,

193 Liszka H, Louwerens JW, Repo JP, Samaila E, Stephens M, Witteveen AGH.

194 EFAS Score - Multilingual development and validation of a patient-reported 
outcome measure (PROM) by the score committee of the European Foot and Ankle Society (EFAS). Foot Ankle Surg 2018; 24(3): 185-204.

197

198 Figure 1a and $\mathrm{b}$. Association between change in EFAS Score from pre- to post-

199 surgery and patient self-reported improvement (a, Finnish; b, Turkish)

200

201 Table 1 Demographic data. $\mathrm{N}=$ sample size; $\mathrm{F}=$ Female; $\mathrm{L} / \mathrm{R} / \mathrm{B}=$

202 Left/Right/Both; N/A = not available

\begin{tabular}{|c|c|c|c|c|}
\hline & $\mathrm{n}$ & $\begin{array}{c}\text { Age } \\
(\mathrm{mean} \pm \mathrm{SD})\end{array}$ & $\begin{array}{c}\text { Sex } \\
(\% \mathrm{~F})\end{array}$ & $\begin{array}{c}\text { Affected side } \\
(\% \mathrm{~L} / \mathrm{R} / \mathrm{B})\end{array}$ \\
\hline Finnish & 130 & $53.8 \pm 15.9$ & 80.0 & $40.0 / 57.7 / 2.3$ \\
\hline Turkish & 131 & $46.9 \pm 14.7$ & 70.0 & $40.8 / 42.1 / 17.1$ \\
\hline
\end{tabular}

204

205

206 Table 2. Prevalence of primary diagnoses, in \%, based on ICD-10 codes

\begin{tabular}{|l|c|c|c|c|}
\hline & $\begin{array}{c}\text { Osteoarthritis } \\
\text { (M19) }\end{array}$ & $\begin{array}{c}\text { Deformities } \\
(\mathrm{M} 20-21, \mathrm{Q} 66)\end{array}$ & $\begin{array}{c}\text { Soft-tissue disorders } \\
(\mathrm{M} 60-79)\end{array}$ & $\begin{array}{c}\text { Other musculoskeletal } \\
(\mathrm{M})\end{array}$ \\
\hline Finnish & 13.8 & 54.0 & 11.7 & 12.3 \\
\hline Turkish & 10.7 & 46.9 & 5.5 & 28.7 \\
\hline
\end{tabular}

208 Table 3. Responsiveness of the EFAS Score.

\begin{tabular}{|l|c|c|}
\hline & Finnish & Turkish \\
\hline Duration of follow up in days: mean (std) & $206(77)$ & $187(39)$ \\
\hline DISTRIBUTION-BASED METRICS & & \\
\hline Effect Size & 0.88 & 1.23 \\
\hline
\end{tabular}




\begin{tabular}{|l|c|c|}
\hline SEM (baseline) & 0.323 & 0.403 \\
\hline$\%$ of patients improving > SEM & 67.7 & 79.4 \\
\hline ANCHOR-BASED METRIC & & \\
\hline $\begin{array}{l}\text { Pearson correlation between change in EFAS-PROM and } \\
\text { patient-reported improvement }\end{array}$ & 0.37 & 0.25 \\
\hline
\end{tabular}

\section{Appendices}

214 Appendix 1, EFAS Score, Finnish version

215 Appendix 2, EFAS Score, Turkish version 